\section{International Scientific Journal Theoretical \& Applied Science}

p-ISSN: 2308-4944 (print) $\quad$ e-ISSN: 2409-0085 (online)

Year: $2018 \quad$ Issue: $01 \quad$ Volume: 57

Published: $30.01 .2018 \quad$ http://T-Science.org

SECTION 33: Advertising technologies.

Creative. Innovations

\section{O. Borduch}

Institute of Service and Entrepreneurship (branch) of DSTU, ( Shakhty, Russia)

\author{
A. V. Golovko. \\ graduate student, \\ Institute of Entrepreneurship \\ and Service sector (branch) DSTU, g. Shakhty \\ V.T. Prokhorov \\ Doctor of technical sciences, professor, \\ professor of the department "Designing, technology \\ and design", ISOP (f) DGTU, g. Shakhty \\ P. N. Kozachenko \\ candidate of chemical Sciences, \\ head of Department "Natural Sciences" \\ Institute of Service and Entrepreneurship \\ (branch) of DSTU, ( Shakhty, Russia)
}

N.V. Tihonova

Doctor of technical sciences, professor, professor of the department «Designing clothing and footwear», Research technological University

(Kazan, Tatarstan

S.M. Zverev

JSC "Roslegprom" in city Moscow Professor, candidate of economic Sciences

(Moscow. Russia)

\title{
NEW IN THE EVALUATION OF THE EFFECTIVENESS OF INNOVATIVE TECHNOLOGICAL SOLUTIONS FOR THE PRODUCTION OF IMPORT-SUBSTITUTING SHOES (2 message)
}

\footnotetext{
Abstract: in the message 2 is the first proposed the dimensionless evaluation index of the competitiveness of innovative technological processes, while ensuring import substitution of light industrial goods for consumers in the regions of SFD and NCFD. The level of competitiveness of innovative technological processes changes from 0 to 1, i.e. failed from bad to effectively high, allowing software to assess the indicators of competitiveness, making adjustments, if not met the conditions for the improvement of effective solutions for enterprises to guarantee their sustained demand and import substitution products in these regions.

Key words: competitiveness, innovative production processes, profit margins, import substitution, dimensionless score, assortment policy, software, stickers, cash flows, price elasticity, production costs.

Language: Russian

Citation: Borduch DO, Golovko AV, Prokhorov VT, Kozachenko PN, Tihonova NV, Zverev SM (2018) NEW IN THE EVALUATION OF THE EFFECTIVENESS OF INNOVATIVE TECHNOLOGICAL SOLUTIONS FOR THE PRODUCTION OF IMPORT-SUBSTITUTING SHOES (2 message). ISJ Theoretical \& Applied Science, 01 (57): 373-385.

Soi: http://s-o-i.org/1.1/TAS-01-57-48 Doi: crostef https://dx.doi.org/10.15863/TAS.2018.01.57.48

UDC 685.34:335.74

НОВОЕ В ОЦЕНКЕ ЭФФЕКТИВНОСТИ ПРИНЯТЫХ ИННОВАЦИОННЫХ ТЕХНОЛОГИЧЕСКИХ РЕШЕНИЙ ДЛЯ ПРОИЗВОДСТВА ИМПОРТОЗАМЕЩАЕМОЙ ОБУВИ (Сообщение 2)
} 
Аннотация: в сообщении 2 впервые предлагается безразмерная оценка показателя конкурентоспособности инновационных технологических прочессов при обеспечении импортозамещчения товаров лёгкой промышленности для потребителей регионов ЮФО и СКФО. Уровень конкурентоспособности инновационных технологических процессов изменяется от 0 до 1 , m. e. от неудачно плохого до эффективно высокого, позволяя с помощзью программного обеспечения оценивать показатели конкурентоспособности, внося коррективы, если не реализованы условия по улучшению эффективных решений для предприятий, чтобы гарантировать им устойчивый спрос и импортозамещение товаров в этих регионах.

Ключевые слова: конкурентоспособность, инновационные технологические процессы, прибыль рентабельность, импортозамещение, безразмерная оценка, ассортиментная политика, программное обеспечение, ценовая ниша, денежные потоки, ценовая эластичность, себестоимость продукиии.

\section{Введение}

Ассортиментная политика заключается в выработке реализации решений относительно номенклатуры (наименований) производимой продукции, разнообразия ассортимента одного наименования, необходимости расширения выпускаемого ассортимента.

Для определения объемов ожидаемого спроса потребителями на новую продукцию и обеспечения сбалансированности между спросом и предложением обувным предприятиям целесообразно использовать метод экспертных оценок. Опрос экспертов (специалистов торговли и промышленности) проводится тогда, когда готовы образцы новой продукции, необходимой для экспертизы[1].

По результатам экспертного опроса составляется итоговый отчет, где определены ожидаемые объемы спроса на продукцию предприятия. На основе этих прогнозных рекомендаций, опроса потребителей и производственных возможностей предприятия составляется оптимальная структура ассортимента.

Один из самых сложных вопросов методологии экспертных опросов - отбор экспертов и формирование комиссии экспертов с наибольшей степенью согласованности мнений и высоким уровнем компетентности.

Уровень компетенции - ключевой критерий протбора экспертов - понятие субъективное, единая методика оценки компетентности экспертов не выработана.
Для формирования оптимальной ассортиментной политики и спроса на продукцию обувного предприятия предлагается использовать одну из методик оценки компетентности экспертов, которая основывается на расчёте коэффициента компетентности Кј.

Коэффициент компетентности $\mathrm{Kj}$ вычисляется на основе суждения эксперта о степени информированности по решаемой проблеме и указания источников аргументации собственного мнения.

Коэффициент компетентности рассчитывается по формуле:

$$
K j=1 / 2 \times(K u j+K a j)
$$

где Киј - коэффициент информированности по проблеме;

Кај - коэффициент аргументации по этой же проблеме.

\section{Основная часть}

Рассмотренный метод оценки компетентности экспертов может быть использован в том случае, если имеется достаточная аргументация о достоверности полученных результатов их работы.

Для обоснованного формирования комиссии эксэкспертов с наибольшей степенью согласованности мнений разработан алгоритм, маматематическое обоснование которого прпредставлено в статье [2].

Данный программный продукт позволяет из имеющейся группы экспертов выделить подгруппу экспертов с наибольшей степенью согласованности мнений (рисунок 1).

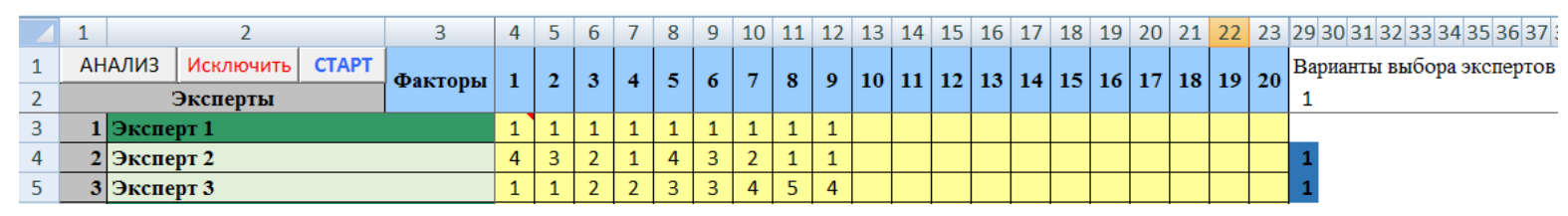

Рис. 1 - Программное обеспечение для оценки согласованности мнений экспертов и их уровня компетентности. 
Также было разработан программный продукт по расчету основных экономических показателей по обувному предприятию.

\section{Данный}

автоматизировать

экономических предприятию.

Для большей наглядности структуры алгоритма расчеты выполняются на отдельных листах Excel по статьям расходов.

Ячейки, выделенные зеленым цветом нужно заполнить исходными данными. Все остальные ячейки расчетных таблиц будут заполнены расчетными данными или данными из справочных татаблиц.

На листе «Производ.прогр.» рассчитывается производственная программа предприятия на год. Задавая планируемые объемы выпуска каждой модели в день, а также ориентировочный срок выпуска каждой модели и стоимость изделия, получаем годовой объем выпуска в натуральном, стоимостном выражении, а также в трудо-часах по каждой модели и по предприятию в целом.

Определяем базовую модель и на листе «Расчет коэф. труд.», задавая рассчитанную технологами трудоемкость единицы изделия в часах по каждой модели, получаем годовой выпуск в трудо-часах по каждой модели, а также коэффициенты трудоемкости каждой модели с учетом выпуска.

На листе «Труд.ресурсы» рассчитываем состав трудового коллектива и баланс рабочего времени одного среднесписочного рабочего на планируемый год.

На листе «Расчет ЗП» выполняем расчет фондов заработной платы.

На листе «Нормы расхода матер.» заполняем таблицы по расходу основных и вспомогательных материалов и получаем стоимость материалов по каждой модели на 100 пар. Общие расходы по основным и вспомогательным материалам сведены в таблицу на листе «Общие матер.».

На последующих листах соответственно выполняются расчеты расходов на топливо и энергию, расходов на содержание и эксплуатацию оборудования, общепроизводственных расходов.

На листе «Себестоимость» выполняется расчет себестоимости на калькуляционную единицу по моделям, а также рассчитываются: оптовая цена, прибыль и ренабельность, затраты на рубль товарной продукции, условнопеременные и условно-постоянные затраты. На этом же листе аналитически рассчитывается точка безубыточности и запас финансовой прочности по каждой модели.

Уровень рентабельности должен быть в пределах от 10 до $25 \%$. По полученным показателям рассчитывается выручка от реализации, валовая выручка с учетом налога на имущество 2,2\% и налога на прибыль в размере $20 \%$, а также чистая прибыль по моделям и по предприятию в целом при условии реализации всего объема произведенной продукции. Образец работы программного продукта приведен на рисунках 9-12.

В современных условиях рыночных отношений, конкурентной среды и непосредственного взаимодействия российских и зарубежных производителей решение проблемы сочетания государственных и рыночных механизмов управления конкурентоспособностью становится стратегическим ресурсом экономики регионов ЮФО и СКФО. В мировой экономике место ценовой конкурентоспособности заняла конкурентоспособность уровней качества, которая повысила свою актуальность с вхождением России в ВТО. Возрастание фактора качества результатов деятельности производства отечественной обуви в стратегии конкурентной борьбы на мировых рынках является долгосрочной тенденцией.

Особенно актуальна задача повышения конкурентоспособности для обувных предприятий, которые в силу внешних факторов (усиление конкуренции вследствие глобализации, мировой финансовый кризис) и внутренних (неэффективный менеджмент) утратили свои конкурентные позиции на внутреннем и внешнем рынках. В ответ на негативные процессы во внешней среде усиливаются процессы регионализации и создания различных сетевых структур, одной из которых является союз товаропроизводителей и государства.

Основой формирования критериев оценки конкурентоспособности предприятий регионов ЮФО и СКФО является содержание понятия «конкурентоспособность предприятия», под которой понимаются его преимущества по сравнению с другими предприятиями в обеспечении экономического развития региона, а также в инновационном и инвестиционном потенциале международного сотрудничества. . Содержание понятия трансформировано в общую модель определения конкурентоспособности предприятия (формула 2).[3]

$$
K_{\Pi \kappa}=f\left(3_{\text {рег }} ; \Pi_{\text {инв }} ; \Pi_{\text {иннов }}\right),
$$

где $K_{П к}-$ оценка конкурентоспособности предприятия ;

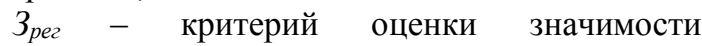
предприятия для экономического развития региона;

$\Pi_{\text {ин }}$ - критерий оценки инвестиционного потенциала предприятия;

$\Pi_{\text {инов }}-$ критерий оценки инновационного потенциала предприятия.

Таким образом, на основе этих критериев конкурентоспособности нами, предложена система показателей оценки значения любого предприятия 
для развития регионов ЮФО и СКФО, которая представлена в таблице 1.

Оценка инновационно-инвестиционного потенциала предприятия. Инновационный потенциал определяется количеством филиалов, входящих в предприятие. Чем больше количество филиалов, тем выше уровень конкуренции, а конкуренция является стимулом для инноваций. Кроме того, чем больше инновационно-активных филиалов в составе предприятия, тем выше инновационный потенцииал самого предприятия.
Инвестициионный потенц̧иал характеризуется количеством уровней переработки продукта в цепочке ценностей. Уровень переработки представляет собой количество видов продуктов, которые создаются на предприятии по производственной цепи, определяемых на основе кода ОКОНХ в соответствии с Классификатором отраслей народного хозяйства .. Чем выше степень переработки продукта, тем больше требуется инвестиций в такое предприятие.

\section{Показатели оценки значимости предприятия для развития регионов ЮФО и СКФО}

\begin{tabular}{|c|c|}
\hline $\begin{array}{l}\text { Направления оценки значения предприятия для } \\
\text { экономики регионов }\end{array}$ & $\begin{array}{l}\text { Показатели оценки значимости } \\
\text { развития регионов }\end{array}$ \\
\hline 1. Содействие росту доходов бюджета & Добавленная стоимость, созданная предприятием \\
\hline 2. Содействие общей занятости & Количество работающих на предприятии \\
\hline $\begin{array}{l}\text { 3. Содействие формированию положительного } \\
\text { сальдо внешней торговли }\end{array}$ & Объем экспорта продукции предприятием \\
\hline $\begin{array}{l}\text { 4. Вклад предприятия в экономику регионов ЮФО } \\
\text { и СКФО }\end{array}$ & $\begin{array}{llll}\text { Доля } & \text { предприятия в } & \text { структуре } & \text { производства } \\
\text { регионов ЮФО и СКФО } & & \end{array}$ \\
\hline
\end{tabular}

Для оценки эффективности разработанных инновационных технологических процессов предлагается использовать коэффициент эффективности $\left(K_{э ф}\right)$, значение которого нужно рассматривать как значение коэффициента конкордации для оценки итогов априорного ранжирования (W), который изменяется от 0 до 1. Если его значение стремится к единице, то это значит, что производителю удалось найти самое оптимальное решение инновационного технологического процесса, если же его значение стремится к нулю - то требуется анализ причин такого неудовлетворительного итога и поиск ошибок, спровоцировавших получение такого результата, и пути устранения допущенных промахов.[4-6]

Коэффициент

эффективности технологического процесса вычисляется по формуле:

$$
\begin{gathered}
K_{\text {э }}=K_{\Pi T} \cdot K{ }^{i} \cdot P_{s} \cdot C \cdot S_{\text {обш }} \cdot 3_{\phi} \\
\cdot T_{\text {б.у. }} \cdot \Pi p \cdot \mathrm{R} \cdot 3_{1 \mathrm{p} \mathrm{тт.}} \cdot 3_{\text {усл.пер.ед }} \cdot 3_{\text {усл.пос.ед.. }}
\end{gathered}
$$

Производительность труда (К Пт)

$$
K_{\Pi T}=\frac{P}{H_{\text {sыp }}},
$$

где $\mathrm{P}$ - задание потока, пар;

$$
H_{\text {выр }} \text { - норма выработки проектная, пар. }
$$

Загрузка рабочих $\left(\mathrm{K}^{\mathrm{i}}\right)$

$$
K_{3}{ }^{i}=\frac{Я c \partial^{P}}{Я c \partial^{\Phi}},
$$

где $Я c \partial^{P}$ - расчетное число рабочих, чел.;

ЯсӘ $\partial^{\Phi}$ - фактическое число рабочих, чел.

Выпуск обуви на $1 \mathrm{~m}^{2}\left(\mathrm{P}_{\mathrm{s}}\right)$

$$
P_{s}=\frac{P}{S_{n p}},
$$

где $S_{n p}$ - площадь производственная, м $^{2}$.

Стоимость оборудования на единицу задания потока (C)

$$
C=\frac{T}{P},
$$

где Т - стоимость оборудования, руб.

Суммарная расценка $\left(\mathrm{S}_{\text {общ }}\right)$

$$
S_{\text {общ }}=\sum_{i=1}^{n} S^{i},
$$

где $S^{i}$ - расценка на і-ой операции; $\mathrm{n}$ - количество операций.

Запас финансовой прочности рассчитывается по следующей зависимости ( $\left.3_{\text {фп }}\right)$

$$
3_{\text {фп }}=\frac{B_{2}-T_{\text {б.y }}}{B_{2}} \cdot 100(\%),
$$

где $\mathrm{B}_{2}$ - выпуск товарной продукции в плановом периоде в натуральном выражении пары;

$\mathrm{T}_{\text {б.у }}$ - точка безубыточности, пары. 


\begin{tabular}{|c|c|c|c|c|c|c|}
\hline Impact Factor: & $\begin{array}{l}\text { ISRA (India) } \\
\text { ISI (Dubai, UAE } \\
\text { GIF (Australia) } \\
\text { JIF }\end{array}$ & $\begin{array}{l}=1.344 \\
=0.829 \\
=0.564 \\
=1.500\end{array}$ & $\begin{array}{l}\text { SIS (USA) } \\
\text { PИНЦ (Russia) } \\
\text { ESJI (KZ) } \\
\text { SJIF (Morocco) }\end{array}$ & $\begin{array}{l}=0.912 \\
=0.207 \\
=4.102 \\
=\mathbf{2 . 0 3 1}\end{array}$ & $\begin{array}{l}\text { ICV (Poland) } \\
\text { PIF (India) } \\
\text { IBI (India) }\end{array}$ & $\begin{array}{l}=6.630 \\
=1.940 \\
=4.260\end{array}$ \\
\hline
\end{tabular}

Точка безубыточности определяется по формуле $\left(\mathrm{T}_{\text {б.y }}\right)$ :

$$
\mathrm{T}_{\text {б.y }}=\frac{3_{\text {усл.пост. }}}{Ц_{\text {ед. }}-3_{\text {усл.пер.еd }} .} \text { (пары), }
$$

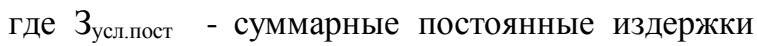
производства единицы производства, руб.;

Цед.- цена единицы продукции, руб.;

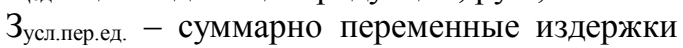
производства единицы производства, руб.

Прибыль единицы продукции определяется по следующей зависимости:

$$
\Pi p .=Ц_{\text {опт }}-\mathrm{C} \text {, }
$$
(отпускная цена за вычетом налога на добавленную стоимость в размере $10 \%$ на детскую обувь и $18 \%$ на другие виды), руб.;

С - полная себестоимость единицы продукции, руб.

Рентабельность продукции (R) определяется по следующей формуле:

$$
\mathrm{R}=\frac{\Pi_{p}}{C} \cdot 100(\%),
$$

где $\Pi_{p}-$ прибыль от реализации единицы продукции, руб.;

С - полная себестоимость единицы продукции, руб.

Затраты на 1 руб. товарной продукции $\left(3_{1 \mathrm{p}}\right.$ т.п.) определяется по следующей формуле:

$$
3_{\text {рр т.п }}=\frac{C}{Ц_{\text {onm }}} \cdot 100 \text { (коп), }
$$

где С - полная себестоимость единицы продукции, руб.

Цопт - оптовая цена единицы продукции (отпускная цена за вычетом налога на добавленную стоимость в размере $10 \%$ на детскую обувь и $18 \%$ на другие виды), руб.

Условно-переменные затраты (суммарные переменные издержки производства единицы продукции) (Зусл. пер.е.) определяется как:

$$
\begin{aligned}
& 3_{\text {усл. пер.ел. }}=\mathrm{C}_{\text {пол }}-(5 \text { ст.с. } . \text { пол }+6 \text { ст.с.ппол. }+7 \\
& \text { ст.с.пол. }+8 \text { ст.с.пол. }+9 \text { ст.с.пол. }) ; \text { (руб. })
\end{aligned}
$$

Условно-постоянные расходы (суммарные постоянные издержки производства единицы продукции) (Зусл. пос.ел.)

$$
\begin{aligned}
& 3_{\text {усл. пос.ед }}=\mathrm{C}_{\text {пол. }}-(1 \text { ст.с. } . \text { пол. }+2 \text { ст.с.пол. }+3 \\
& \text { ст.с.пол. }+4 \text { ст.с.пол.); (руб.) }
\end{aligned}
$$

Также было разработано программное обеспечение для выбора оптимальной мощности

При этом в качестве критериев для обоснованного выбора оптимальной мощности при формировании алгоритма оправданно были выбираны именно те критерии, которые оказывают наибольшее влияние на себестоимость готовой продукции, а именно:

$\square$ потери по заработной плате на единицу продукции, руб.;

$\square$ выпуск обуви, $1 \mathrm{~m}^{2}$;

$\square$ процент загрузки рабочих, \%;

производительность труда одного

рабочего, пары;

удельные приведенные затраты на 100 пар обуви, руб.;

$\square$ стоимость оборудования на единицу задания потока (C)

суммарная расценка (Sобщ);

запас финансовой прочности (Зфп);

точка безубыточности (Тб.у);

прибыль единицы продукции (Пр.);

рентабельность продукции (R);

(31р т.п.)

затраты на 1 руб. товарной продукции

пер.ед.);

условно-переменные затраты (Зусл.

Из приведенных критериев, по-нашему мнению, производитель имеет возможность отдать предпочтение тем, которые с его точки зрения гарантировали бы ему производство импортозамещаемой, конкурентоспособной и востребованной продукции, а именно:[7-9]

- производительность труда 1 рабочего важнейший трудовой показатель. От уровня и динамики производительности труда зависят в той или иной степени все основные показатели эффективности производства и все трудовые показатели: производство продукции, численность работников, расходование заработной платы, уровень оплаты труда и т. д., для повышения производительности труда первостепенное значение имеют внедрение новой техники и технологии, широкая механизация трудоемких работ, автоматизация производственных процессов, повышение квалификации рабочих и служащих, особенно при внедрении инновационных технологических процессы на базе универсального и многофункционального оборудования;

- удельные приведенные затраты показатель сравнительной экономической эффективности капитальных вложений, применяемый при выборе лучшего из вариантов решения технологических задач.;

- приведенные затраты - сумма текущих затрат, учитываемых в себестоимости продукции, и единовременных капитальных вложений, сопоставимость которых с текущими затратами достигается путем умножения их на 
нормативный коэффициент эффективности капитальных вложений;

- запас финансовой прочности (Зфп) показывает, на сколько процентов предприятие может снизить объём реализации, не неся убытков;

- точка безубыточности позволяет (Тб.у) определить тот минимально необходимый объём реализации продукции, при котором предприятие покрывает свои расходы и работает безубыточно, не давая прибыли, но и не терпит убытков, то есть это минимальный размер выпуска продукции, при котором достигается равенство доходов от продаж и издержек производств;

- прибыль (убыток) от реализации продукции (Пр) определяется как разница между выручкой от реализации продукции в действующих ценах НДС и акцизов и затратами на её производство и реализации;

- рентабельность продукции (R) отражает зависимость между прибылью от реализации единицы продукции и её себестоимостью;

- условно-постоянные расходы (суммарные постоянные издержки производства единицы продукции) (Зусл.пос.ед), которые изменяются пропорционально или почти пропорционально изменению объёма производства (1ст - затраты на сырьё и материалы; 2ст - затраты на вспомогательные материалы; 3cт - затраты на топлива и энергии на технологические нужды; 4 ст - затраты на дополнительную и основную зарплаты производственных рабочих со страховыми взносами во внебюджетные фонды);

- условно-переменные расходы (суммарные переменные издержки производства единицы продукции) (Зусл. пер.ед.), которые не зависят или почти не зависят от изменения объёма производства (5ст - затраты на расходы на подготовку и освоение производства; 6 ст затраты на расходы на содержание и эксплуатацию оборудования; 7ст - затраты на общепроизводственные нужды; 8cт - затраты на общехозяйственные расходы, они вместе с условно-постоянными расходами составляют производственную себестоимость; 9 ст - затраты на коммерческие расходы. Все эти статьи формирующие условно-переменные и расходы и условно-постоянные расходы составляют полную себестоимость, то есть условно-переменные расходы могут определяться как полная себестоимость - условно-постоянные расходы, и наоборот, условно постоянные расходы могут определяться как полная себестоимость условно-переменные расходы);

- затраты на 1 руб. товарной продукции показывают относительный размер прибыли на каждый рубль текущих расходов, то есть это отношение себестоимости единицы продукции к оптовой цене, характеризующая эффективность проведённых мероприятий по повышению конкурентоспособности и востребованности продукции на рынках спроса.

Для перевода размерных оценок показателей в безразмерные предлагается использовать индексный метод. Индексы безразмерных показателей определяют по формуле (16) для nозитивных показателей, имеющих положительную тенденцию - рост (например, рентабельность реализованной продукции, производительность труда) и по формуле (17) для негативных показателей, имеющих положительную тенденцию - снижение (например, износ основных средств, превышение остатков готовой продукции на складе по сравнению с нормой, коэффициент текучести кадров), взятые, в основном, из показателей, формирующих себестоимость продукции:

$$
\begin{gathered}
O_{i}=X_{i} / X_{i}^{\max } \\
O_{i}=X_{i}^{\min } / X_{i}
\end{gathered}
$$

где $O_{i}-$ безразмерная (индексная) оценка і-го показателя конкурентоспособности предприятия,

$X_{i}$ - значение і-го размерного показателя оценки конкурентоспособности предприятия,

$X_{i}^{\max }$ - максимальное значение і-го размерного показателя оценки конкурентоспособности предприятия,

$X_{i}^{\text {min }}$ - минимальное значение і-го размерного показателя оценки конкурентоспособности предприятия.

Этап 4. Оценка конкурентоспособности товара. Осуществляется для товаров легкой промышленности по их спросу на внутреннем рынке.

Этап 5. Расчет обобщаюмего показателя конкурентоспособности предприятия. Количественную оценку конкурентоспособности предприятия предлагается определять по следующей формуле (18).

$$
K_{\Pi}=\sum_{i=1}^{m} \alpha_{i} \times O_{i}
$$

где $K_{\Pi}-$ оценка конкурентоспособности предприятия в процентах,

$\alpha_{i} \quad-$ значимость і-го показателя конкурентоспособности в процентах,

$O i$ - индексная (безразмерная) оценка і-го показателя конкурентоспособности,

$m$ - количество показателей оценки конкурентоспособности предприятия. 
Значения оценки конкурентоспособности предприятия теоретически могут изменяться в пределах от 0 до 100 (соотношение 19).

$$
K_{n}=0 \div 100
$$

Для качественной характеристики полученных оценок конкурентоспособности необходима шкала оценки качественного уровня. В экономической практике используют принцип построения шкал с равным шагом, прогрессивные и регрессивные шкалы. Прогрессивные и регрессивные шкалы чаще всего используют для материального стимулирования. Полагаем, что наиболее целесообразной является шкала с равным шагом, поскольку она, во-первых, соответствует решению практической задачи (спецификации качественного уровня конкурентоспособности), во-вторых, проста в построении и использовании. Шаг шкалы определяется как 100 (максимальная оценка): 4 (количество уровней $)=25$. Возможен выбор и другого значения шага, что определяется целями и задачами, которые формирует себе само предприятие. В результате расчета была получена следующая шкала оценки качественного уровня конкурентоспособности предприятия (таблица 2)

\section{Шкала оценки качественного уровня конкурентоспособности предприятия}

Таблица 2

\begin{tabular}{|l|l|}
\hline Оценка в процентах & Качественный уровень \\
\hline от 0 до 24,9 & очень низкий \\
\hline от 25,0 до 49,9 & низкий \\
\hline от 50,0 до 74,9 & средний \\
\hline от 75,0 до 100 & высокий \\
\hline
\end{tabular}

$$
\mathrm{K}_{\ni \phi}=\mathrm{K}_{1} \cdot \mathrm{K}_{2} \cdot \mathrm{K}_{3} \cdot \mathrm{K}_{4} \cdot \mathrm{K}_{5} \cdot \mathrm{K}_{6} \cdot \mathrm{K}_{7} \cdot \mathrm{K}_{8} \cdot \mathrm{K}_{9} \cdot \mathrm{K}_{10} \cdot \mathrm{K}_{11} \cdot \mathrm{K}_{12} \text {, }
$$

где Кэф - коэффициент весомости оценки эффективности инновационных технологических процессов, сформированные для производства конкурентоспособной и востребованной продукции (ПТ);

$K_{1}$ - весомость производительности труда

$\mathrm{K}_{2}$ - весомость загрузки рабочих ( $3_{\mathrm{P}}$ );

$\mathrm{K}_{3}$ - весомость выпуска обуви $\left(\mathrm{P}_{\mathrm{s}}\right)$;

$\mathrm{K}_{4}$ - весомость стоимости оборудования на единицу задания потока (C);

$\mathrm{K}_{5}$ - весомость суммарной расценки на единицу продукции $\left(\mathrm{S}_{\text {обш }}\right)$;

$\mathrm{K}_{6} \quad$ - весомость запаса финансовой прочности ( $\left.3_{\text {фп }}\right)$;

$\mathrm{K}_{7}$ - весомость точки безубыточности $\left(\mathrm{T}_{\text {б.y }}\right)$;

$\mathrm{K}_{8}$ - весомость прибыли единицы продукции (Пр.); (R)

$\mathrm{K}_{9}$ - весомость рентабельности продукции

$\mathrm{K}_{10}$ - весомость затрат на 1 рубль товарной продукции ( $\left.3_{1 \text { р.т. }}\right)$;

$\mathrm{K}_{11}$ - весомость условно-переменных затрат (суммарные переменные издержки производства

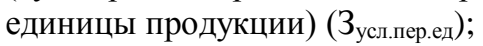

$\mathrm{K}_{12}$ - весомость условно-постоянных расходов (суммарные постоянные издержки производства единицы продукции) (Зуслпос.ед)

Себестоимость услуг и продукции - это текущие затраты предприятия на производство и реализацию услуг и продукции, выраженные в денежной форме. При исчислении себестоимости услуг и продукции все расходы предприятия классифицируются по различным признакам:

- в зависимости от характера отнесения их на себестоимости услуг и продукции разделяют на 2 группы: прямые и косвенные.

Прямыми называются такие расходы, которые могут быть непосредственно отнесены на тот или иной вид продукции при выработке более одного ее вида (материалы, топливо, энергия).

Косвенные - расходы, которые не могут быть непосредственно отнесены на себестоимость различных видов продукции при изготовлении и ремонте более одного ее вида, а далее распределяется между ними пропорционально другим затратам средств или труда.

- в зависимости от изменения объема производства все расходы подразделяются на условно-переменные (пропорциональные) и условно-постоянные (непропорциональные).

К условно-переменным относятся расходы, которые изменяются пропорционально или почти пропорционально изменению объема производства (затраты на материалы и энергию на технологические цели, заработная плата производственных рабочих и др.).

K условно-постоянным относятся расходы, которые не зависят или почти не зависят от изменения объема производства (амортизационные отчисления от стоимости 
основных фондов, аренда, расходы на содержание зданий и сооружений, заработная плата руководителей, специалистов и служащих и т.п.):

- по экономической роли в процессе производства: основные и накладные;

- по составу (однородности):

одноэлементные, комплексные;

- по периодичности возникновения: текущие и единовременные.

Единовременные - затраты на подготовку и освоение производства новых видов продукции, расходы, связанные с пуском новых производств и другое:

- по участию в процессе производства: производственные и коммерческие;

- по эффективности: производительные, непроизводительные.

Производительными считаются затраты на производство продукции установленного качества при рациональной технологии и организации производства.

Непроизводительные расходы являются следствием недостатков в технологии организации производства (потери от простоев, брак продукции, оплата сверхурочных и др.).

Производительные расходы планируются, а непроизводительные не планируются.

Калькулированием себестоимости услуг и продукиии называется определение себестоимости вырабатываемой продукции и оказываемых услуг, проводимое по отдельным статьям затрат. Расчет себестоимости при калькулировании производится на типовые калькуляционные единицы.

Плановые калькуляции составляются по номенклатуре калькуляционных статей:

1. Сырье и основные материалы (с учетом транспортно-заготовительных расходов и за вычетом реализуемых отходов).

2. Вспомогательные материалы.

3. Топливо и электроэнергия на технологические цели.

4. Основная и дополнительная заработная плата производственных рабочих со страховыми взносами во внебюджетные фонды.

5. Расходы на подготовку и освоение производства.

6. Расходы на содержание и эксплуатацию оборудования (РСЭО).

7. Общепроизводственные расходы (цеховые расходы).

8. Общехозяйственные расходы.

9. Платежи по обязательному страхованию имущества.

10. Производственная себестоимость
11. Коммерческие

расходы.

Полная себестоимость

Смета затрат на производство $u$ финансовые результатьл

Для определения общей суммы всех плановых затрат на предприятии и взаимной увязки показателей себестоимости, прибыли и рентабельности с другими показателями составляют смету затрат на производство по экономическим элементам, в которую включают расходы всех структурных подразделений предприятия, участвующих в выполнении услуг (изготовление продукции).

Сметой затрат считается сводный документ, характеризующий денежное выражение всех материальных, энергетических затрат, необходимых для обеспечения выполнения плана по выпуску продукции и услуг.

Расходы, учитываемые в смете, группируются следующим образом.

Смета расходов

1. Сырье и основные материалы.

2. Вспомогательные материалы.

3. Покупные изделия и полуфабрикаты.

4. Топливо со стороны.

5. Энергия со стороны.

6. Основная и дополнительная заработная плата промышленно-производственного персонала (ППП) с отчислениями на единый социальный налог.

7. Амортизация основных фондов на полное восстановление.

8. Прочие расходы.

Формирование финансовых результатов. Конечный финансовый результат (прибыль или убыток) слагается из финансового результата от реализации продукции (работ, услуг), основных средств и иного имущества предприятия и доходов от внереализационных операций, уменьшенных на сумму расходов по этим операциям.

Прибыль (убыток) от реализации продукции (работ, услуг) и товаров определяется как разница между выручкой от реализации продукции (работ, услуг) в действующих ценах без НДС и акцизов и затратами на ее производство и реализацию.

Плановая прибыль $\left(\Pi_{n л}\right)$ :

$$
\Pi_{\text {пл }}=(\text { В } \cdot \text { Ц })-(B \cdot \mathrm{C}),
$$

$\mathrm{B}$ - выпуск товарной продукции в плановом периоде в натуральном выражении;

Ц - цена за 1 пару обуви (единицу продукции) за вычетом НДС и акцизов - это оптовая цена.

С - себестоимость полная единицы продукции.

Прибыль 1 пары $\left(\Pi_{1}\right)$ : 


\begin{tabular}{|c|c|c|c|c|c|c|}
\hline Impact Factor: & $\begin{array}{l}\text { ISRA (India) } \\
\text { ISI (Dubai, UAE } \\
\text { GIF (Australia) } \\
\text { JIF }\end{array}$ & $\begin{array}{l}=1.344 \\
=0.829 \\
=0.564 \\
=1.500\end{array}$ & $\begin{array}{l}\text { SIS (USA) } \\
\text { PИНЦ (Russia) } \\
\text { ESJI (KZ) } \\
\text { SJIF (Morocco) }\end{array}$ & $\begin{array}{l}=0.912 \\
=0.207 \\
=4.102 \\
=\mathbf{2 . 0 3 1}\end{array}$ & $\begin{array}{l}\text { ICV (Poland) } \\
\text { PIF (India) } \\
\text { IBI (India) }\end{array}$ & $\begin{array}{l}=6.630 \\
=1.940 \\
=4.260\end{array}$ \\
\hline
\end{tabular}

$$
\Pi_{1}=L_{o n}-C^{1},
$$

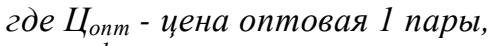

$\mathrm{C}^{1}$ - себестоимость 1 nары.

Рентабельность продукиии отражает зависимость между прибылью от реализации продукиии и её себестоимости.

Она показывает относительный размер прибыли н каждый рубль текущчих расходов и определяется по формуле:

$$
R_{n}=\frac{\Pi_{p}}{3} \cdot 100,
$$

где $R_{n}$ - рентабельность продукичии;

$\Pi_{p}-$ прибыль от реализации продукции;

3-затраты (себестоимость).

$$
R=\frac{\Pi}{C / C} \cdot 100(\%) \text {-расчет на } 1 \text { napy. }
$$

Выручка от реализации продукции (работ и услуг) определяется либо по мере ее оплаты, либо по мере отгрузки товаров (выполнение работ, услуг) и предъявления покупателю (заказчику) расчетных документов.

\section{К доходам относятся:}

- доходы, полученные на территории РФ и за ее пределами от долевого участия в деятельности других предприятий, дивиденды по акциям и доходы по облигациям и другим ценным бумагам, принадлежащим предприятию;

- доходы от сдачи имущества в аренду;

- доходы от до оценки производственных запасов и готовой продукции;

- присужденные или признанные должниками штрафы, пени, неустойки и другие виды санкций за нарушение условий хозяйственных договоров, а также доходы от возмещения причиненных убытков;

- прибыль прошлых лет, выявленная в отчетном году;

- другие доходы от операций, непосредственно связанных с производством и реализацией продукции (работ и услуг).

К расходам и потерям относятся:

- затраты законсервированных возмещаемых за счет других источников);

- не компенсируемые виновниками потери от простоев по внешним причинам;

- потери от уценки производственных запасов и готовой продукции;

- убытки по операциям с тарой;

- судебные издержки и арбитражные расходы;

- присужденные или признанные штрафы, пени, неустойки и другие виды санкций за нарушение условий хозяйственных договоров, а также расходы по возмещению причиненных убытков;

- убытки прошлых лет, выявленных в текущем году;

- не компенсируемые убытки в результате пожаров, аварий, других чрезвычайных ситуаций, вызванных экстремальными условиями; не компенсируемые потери от стихийных бедствий (уничтожение и порча производственных запасов готовых изделий и других материальных ценностей, потери от остановки производства и прочее), включая затраты, связанные с ликвидацией последствий стихийных бедствий; убытки от хищений, виновники которых по решениям суда не установлены.

Анализ безубыточности позволяет определить тот минимально необходимый объем реализации продукции, при котором предприятие покрывает свои расходы и работает безубыточно, не давая прибыли, но и не терпит убытков.

В самом общем виде деятельность любого предприятия осуществляется по схеме «затраты - процесс производства - прибыль».

Точка безубыточности $\left(\mathrm{T}_{\sigma} / \mathrm{y}\right)$ определяется расчетом по следующей формуле

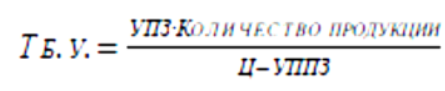

где УПЗ - условно-постоянные затраты на единицу продукции, руб.; УППЗ - условнопеременные затраты на единицу продукции, руб.; Ц - цена единицы продукции без НДС, руб.

Для построения графика безубыточности следует составить уравнение следующего вида:

$$
\begin{aligned}
& \mathrm{y}_{1}=a x ; \\
& y_{2}=a o+a x
\end{aligned}
$$

где $\mathrm{y}_{1}$ - выручка, руб; у2 - затраты (полная себестоимость) на производство продукции, руб.; $а$ - цена единицы продукции без НДС, руб.; $\mathrm{x}$ - планируемый объём реализации продукции, пар; а 0 - сумма УП3; $a_{1}$ - сумма УППЗ на единицу продукции, руб.

Запас финансовой прочности (Зф) показывает насколько можно снизить объём производства, работая безубыточно:

$$
3 \phi=\frac{B-T \tilde{o} \cdot y}{B} \cdot 100(\%),
$$

где Т б.у. - точка безубыточности 


\begin{tabular}{|c|c|c|c|c|c|c|}
\hline Impact Factor: & $\begin{array}{l}\text { ISRA (India) } \\
\text { ISI (Dubai, UAE } \\
\text { GIF (Australia) } \\
\text { JIF }\end{array}$ & $\begin{array}{r}=1.344 \\
=0.829 \\
=0.564 \\
=1.500\end{array}$ & $\begin{array}{l}\text { SIS (USA) } \\
\text { PИHЦ (Russia) } \\
\text { ESJI (KZ) } \\
\text { SJIF (Morocco) }\end{array}$ & $\begin{array}{l}=0.912 \\
=0.207 \\
=4.102 \\
=\mathbf{2 . 0 3 1}\end{array}$ & $\begin{array}{l}\text { ICV (Poland) } \\
\text { PIF (India) } \\
\text { IBI (India) }\end{array}$ & $\begin{array}{l}=6.630 \\
=1.940 \\
=4.260\end{array}$ \\
\hline
\end{tabular}

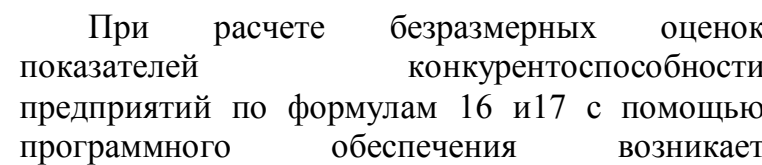
программного обеспечения
необходимость сформулировать эти самые критерии в качестве их доказательной базы. Так, например, прибыль единицы продукции рассчитывается в зависимости от рентабельности продукции, то есть, сначала формулируется размер рентабельности от $5 \%$ до $25 \%$, а потом закладывается размер прибыли единицы продукции. Такая же особенность существует с определением критерия производительности труда, потому что сначала используют инновационные технологические процессы , сформированные на основе универсального и многофункционального оборудования, обслуживание которым должно доверяться высококвалифицированным и ответственным исполнителям, сопереживающие за общий результат работы всего технологического цикла, гарантирующий им производство востребованной и конкурентоспособной продукции , пользующаяся у потребителей отечественных рынков повышенным спросом. Расчёт условнопостоянных расходов на производство единицы продукции и условно-переменных затрат на производство единицы продукции взаимосвязан с особенностями организации производства конкурентоспособной и востребованной продукции, в том числе и для детей. Анализ результатов деятельности ведущих зарубежных производителей подтверждает тот факт, что если условно-постоянные расходы составляют $20 \%$ $40 \%$ себестоимости продукции, то, естественно, условно -переменные затраты - $60 \%$ - 80\%.При этом, вновь необходимо заострить внимание на особенность производства продукции для детей, когда и прибыль, рентабельность, условно- постоянные расходы и условно-переменные затраты формируются на основе реализации требований технических регламентов и нормативных документов и актов , гарантирующие им при их использовании безопасность жизни. И если это обусловлено необходимостью их производства с такими жесткими характеристиками - государство и производители обязаны быть заинтересованными друг в друге и предусматривать производителям компенсацию за дополнительные затраты на их соблюдение и гарантию того, что изготовленная продукция не принесет вред здоровью детям.

Конечно, если критерий по потере зарплаты на единицу продукции должен стремиться к нулю, а объем выпуска обуви с $1 \mathrm{~m}^{2}-$ к его максимально возможному значению, а затраты на 1 рубль товарной продукции должны стремиться к их минимально возможному значению и стоимость оборудования на единицу задания потока тоже стремиться к своему минимально возможному значению, а другие критерии - к их максимально возможному значению- в совокупности безразмерная оценка эффективности разработанных инновационных технологических процессов $(K)$ должна всегда стремиться к единице и этим самым подтверждать всегда, что спроектированный инновационный технологический процесс предприятию для производства им импортозамещаемой продукции будет успешным в своей деятельности на благо населения тех регионов, где они будут функционировать , являясь для этих малых средних городов градообразующими и в которых заинтересованы все ветви властей - как федеральные, так региональные и м муниципальные.

Таблица 3

Расчёт технико-экономических показателей при производстве мужской/женской обуви с диапазоном 300-900 пар в смену

\begin{tabular}{|c|c|c|c|c|c|c|}
\hline 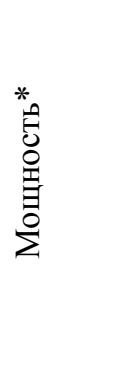 & 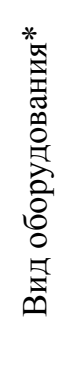 & 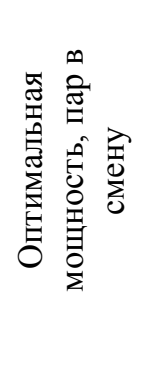 & 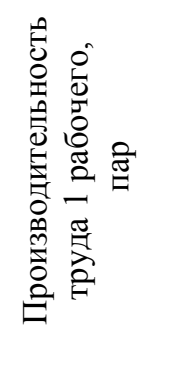 & 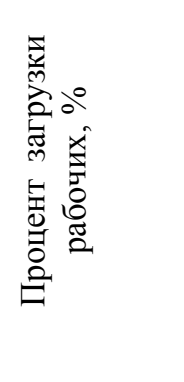 & 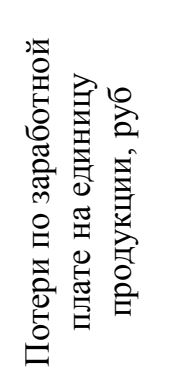 & 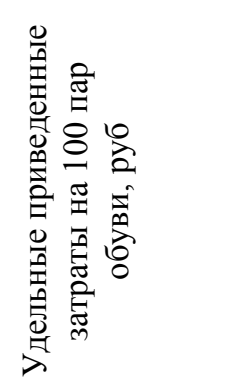 \\
\hline $300-500$ & 1 & $500 / 500$ & $28,09 / 27,73$ & $61,39 / 62,18$ & $13,68 / 13,4$ & $6735,36 / 6980,5$ \\
\hline $500-700$ & 1 & $556 / 700$ & $27,73 / 27,73$ & $69,14 / 69,14$ & $9,83 / 9,83$ & $6404,71 / 6277,43$ \\
\hline
\end{tabular}




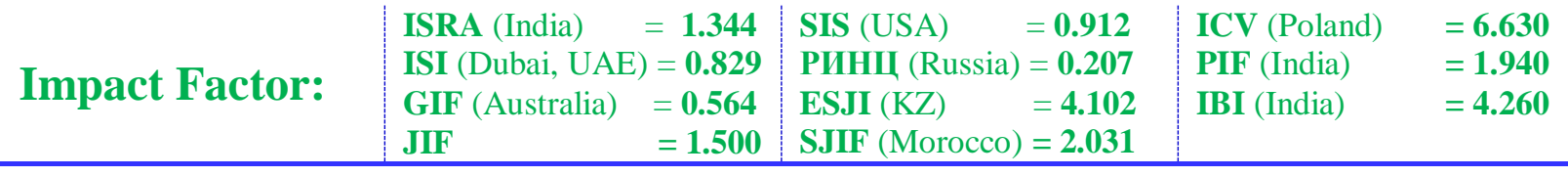

\begin{tabular}{|l|l|l|l|l|l|l|}
\hline $700-900$ & 1 & $889 / 847$ & $28,09 / 27,73$ & $77,20 / 74,5$ & $6,42 / 7,54$ & $5236,17 / 6277,43$ \\
\hline $300-500$ & 2 & $500 / 500$ & $28,09 / 24,45$ & $61,39 / 63,9$ & $13,68 / 14,01$ & $6728,68 / 7630,92$ \\
\hline $500-700$ & 2 & $556 / 556$ & $27,91 / 27,73$ & $68,70 / 69,14$ & $9,97 / 9,83$ & $6083,28 / 6404,71$ \\
\hline $700-900$ & 2 & $889 / 812$ & $28,09 / 25,64$ & $77,20 / 75,4$ & $6,42 / 7,77$ & $5240,72 / 6060,55$ \\
\hline $300-500$ & 3 & $500 / 500$ & $28,09 / 27,0$ & $61,39 / 61,74$ & $13,68 / 14,02$ & $7533,95 / 7827,12$ \\
\hline $500-700$ & 3 & $700 / 556$ & $28,12 / 29,32$ & $67,28 / 68,2$ & $10,56 / 9,71$ & $6734,02 / 6607,65$ \\
\hline $700-900$ & 3 & $889 / 847$ & $28.09 / 27,0$ & $77,20 / 74,7$ & $6,42 / 7,66$ & $5876,59 / 6341,05$ \\
\hline
\end{tabular}

* - варианты мощности и виды оборудования

Характеристика конкурентных преимуществ при производстве всего ассортиментного ряда обуви для принятия решения о его изготовлении, рассчитанный с использованием этого же программного продукта, приведена в таблице 4.

Калькуляционные составляющие для всего ассортиментного ряда.

Таблица 4

\begin{tabular}{|c|c|c|c|c|c|}
\hline \multirow{2}{*}{ Показатели } & \multirow{2}{*}{ Род обуви } & \multicolumn{4}{|c|}{ Виды обуви } \\
\hline & & Весна & Лето & Осень & Зима \\
\hline \multirow{3}{*}{$\begin{array}{c}\text { Себестоимость } \\
\text { единицы } \\
\text { продукции,руб. }\end{array}$} & Мужская & 856,77 & 643,72 & 998,5 & 1007,07 \\
\hline & Женская & 933,51 & 844,31 & $\begin{array}{l}1062,3 \\
7\end{array}$ & 2107,29 \\
\hline & Детская & 551,05 & 503,89 & 586,15 & 795,41 \\
\hline \multirow{3}{*}{$\begin{array}{c}\text { Затраты на } \\
\text { основные } \\
\text { материалы,руб. }\end{array}$} & Мужская & 541,61 & 378,64 & 623,16 & 660,42 \\
\hline & Женская & 523,71 & 511,6 & 618,52 & 1503,57 \\
\hline & Детская & 235,78 & 200,05 & 280,76 & 415,5 \\
\hline \multirow{3}{*}{$\begin{array}{l}\text { Затраты на } \\
\text { вспомогательные } \\
\text { материалы, руб. }\end{array}$} & Мужская & 23,82 & 17,57 & 28,16 & 30,4 \\
\hline & Женская & 22,65 & 17,05 & 24,31 & 43,16 \\
\hline & Детская & 11,78 & 7,92 & 12,16 & 15,26 \\
\hline \multirow{3}{*}{$\begin{array}{c}\text { Заработная } \\
\text { плата }\end{array}$} & Мужская & 141,02 & 108,28 & 161,1 & 150,71 \\
\hline & Женская & 148,92 & 84,62 & 139,09 & 220,58 \\
\hline & Детская & 58,44 & 55,42 & 68,95 & 95,77 \\
\hline \multirow{3}{*}{$\begin{array}{c}\text { Рентабельность } \\
\text { единицы } \\
\text { продукции, руб. }\end{array}$} & Мужская & 10,75 & 14,65 & 13,36 & 15,12 \\
\hline & Женская & 11,88 & 13,37 & 16,42 & 17,11 \\
\hline & Детская & $\mathbf{9 , 5 3}$ & 8,39 & 9,19 & 10,72 \\
\hline \multirow{3}{*}{$\begin{array}{c}\text { Затраты на } 1 \text { руб. } \\
\text { товарной } \\
\text { продукции, руб. }\end{array}$} & Мужская & 82,88 & 85,35 & 86,64 & 84,88 \\
\hline & Женская & 88,12 & 86,63 & 83,57 & 82,89 \\
\hline & Детская & 90,47 & 91,62 & 90,8 & 89,28 \\
\hline
\end{tabular}

Таким образом, разработанное авторами программное обеспечение для оценки эффективности сформированных инновационных технологических процессов для производства импортозамещаяемого ассортимента обуви с учетом рассчитанных калькуляционных составляющих позволяет принять оправданное решение по его запуску, решение о его сбалансированности, гарантированный спрос и обеспечение предприятию устойчивое финансовое положение.

Кроме того, разработанное программное обеспечение позволит региональным и муниципальным ветвям власти совместно с будущими производителями всего ассортиментного ряда обуви в моногородах сформировать объёмы выпуска обуви не только с учетом их востребованности, но и гарантировать предприятиям их устойчивое финансовое 
положение за счет обеспечения себе стабильных ТЭП, то есть будут созданы условия для формирования новых рабочих мест с одновременным решением для них всех социальных проблем, характерные сегодня большинству таким малым и средним городам в других, более благополучных, регионах РФ.

При этом, необходим выбор таких технологических решений, чтобы эффективно реализовывать намеченные цели, обеспечивая предприятиям гарантию того, что разработанный ими ассортимент обуви будет реализован в полном объёме и позволит получать им максимальную прибыль.

Для решения данной задачи необходимо широко использовать литьевой метод, который обеспечивает изготовление всего ассортиментного ряда обуви высокого качества при эффективной рентабельности всех видов обуви при удовлетворении спроса всех групп населения.[7-9]

В затратах на производство обуви наибольший удельный вес составляют расходы на сырье и основные материалы, а затем на заработную плату и амортизационные отчисления.

Авторы считают, что преимущества прямого литья низа обуви несомненно заинтересуют производителей выпускать такой ассортимент, который не только будет отвечать направлениям моды, но что особенно важно - удовлетворять спрос с учетом их функциональных требований к самой обуви, а именно, для спортсменов, для отдыха, для пожилых, для людей с незначительными патологическими отклонениями стопы, создавая им комфортные условия и удовлетворяя спрос на нее, покрывая дефицит за счет варьирования цены на нее.

Одно из условий конкурентоспособности предприятия - организация эффективного взаимодействия с заинтересованными в успешном функционировании этого предприятия сторонами. У каждого предприятия, даже у небольших есть несколько групп субъектов с разными интересами, с которыми оно может находиться во временном или постоянном сотрудничестве. Вопросам изучения этих интересов, путям решения возникающий проблем между внешними и внутренними участниками, налаживанию взаимоотношений между партнерами, посвящены исследования авторов, чтобы гарантировать всем заинтересованным сторонам реализацию главного принципа интересы всех сторон законны и требуют их удовлетворения и уважительного отношения.

Предложенная методика оценки и анализа конкурентоспособности предприятия, в отличие от существующих, во-первых, учитывает специфику отрасли «легкая промышленность», во-вторых, снижает субъективный фактор, втретьих, позволяет проводить эффективный углубленный анализ, чтобы успешно реализовывать достижение сформулированных задач.

\section{References:}

1. Prokhorov V. T., Maltsev I. M. (2004) Software for screening factors in the active conduct of the experiment. The program for computer №2004611762729. Registered in the Register of computer programs 26.07.2004

2. (2007) The software to solve the problem of non-stationary processes of heat exchange systems of the "Stop - footwear environment", provided the dependence of conductivity on temperature: the certificate of official registration of computer programs № 2008610087 / Mikhailov A. B. Was 09.01.2008. - Registration in the register of computer programs. Application No. 2007614069 (17.10.07).
3. (2009) Software the task of assessing the comfortable stay of man in shoes depending on the changes in heat flux stop time-a certificate of official registration of computer programs №2009613371 / Aspen T. M. Was 26.06.2009. -Registration in the register of computer programs. Application No. 2009611957 (29.04.09).

4. Aspen T. M., et al. (2011) the Certificate of official registration of computer programs №2011611394. Software for calculation of the temperature field of the unsteady heat transfer process in the "Stop - footwear - environment" when exposed to stop low temperatures. Issued by the Russian Agency for patents and trademarks (ROSPATENT) 11.02.2011 g. 


\begin{tabular}{l|lr|ll|ll} 
& ISRA (India) & $=\mathbf{1 . 3 4 4}$ & SIS (USA) & $=\mathbf{0 . 9 1 2}$ & ICV (Poland) & $=\mathbf{6 . 6 3 0}$ \\
Impact Factor: & ISI (Dubai, UAE) $=\mathbf{0 . 8 2 9}$ & PUHЦ (Russia) $=\mathbf{0 . 2 0 7}$ & PIF (India) & $=\mathbf{1 . 9 4 0}$ \\
& GIF (Australia) & $\mathbf{0 . 5 6 4}$ & ESJI (KZ) & $=4.102$ & IBI (India) & $=\mathbf{4 . 2 6 0}$ \\
& JIF & $=\mathbf{1 . 5 0 0}$ & SJIF (Morocco) & $=\mathbf{2 . 0 3 1}$ & & \\
\hline
\end{tabular}

5. Aspen T. M., et al. (2011) the Certificate of official registration of computer programs №2011619212. Software description the local unsteady heat transfer in the system of "Stopfootwear-environment" for different climate zones". Issued by the Russian Agency for patents and trademarks (ROSPATENT) 30.11.2011

6. Shrivel I. S., Maltsev I. M., Prokhorov V. T., korablina S. Y., ASP, T. M, Kushnareva I. A. (2015) On a new method for assessing the competence of experts for work in the customs examination // "Science and technology in the modern world: traditions and innovations" proceedings of II All-Russian scientificpractical conference with international participation, 19-20 November 2015: Novosibirsk, 2015. - p. 123-132.

7. Reva D. V., Maltsev I. M., Volkova G. Yu., Prokhorov V. T., S. Yu. Polukhina, Osina T. M. (2015) About the possibilities of new assessment tools to assess the quality of training of highly qualified specialists // the Collection "the role of the modern University technical personnel and modernization of the Russian economy collected works of the IX International scientific.-method. Conf.. Kostroma state technological University" / Kostroma, 2015. -p. 226-231.

8. Reva D. V., Shrivel I. S., Prokhorov V. T., Duyun, L. V., Maltsev, I. M., ASP, T. M. (2015) New possibilities of expert estimations as an instrument of evaluation of quality of preparation of experts // the Collection "the role of the modern University technical personnel and modernization of the Russian economy collected works of the IX International scientific.-method. Conf.. Kostroma state technological University" / Kostroma, 2015. p. 22-28.

9. (2014) The quality revolution: through the ad or through a quality real: monograph V. T. Prokhorov [et al.]; under the General editorship of doctor of technical Sciences, Professor V. T. Prokhorov; VoIP (branch) of DSTU. Novocherkassk: URGU (NPI), 2014. - 384 p. 